\title{
Droit de la famille originairement africain : une analyse critique du pluralisme successoral en République Démocratique du Congo
}

\author{
Par NKUMISONGO Robert* et KALALA ILUNGA Matthiesen**
}

\section{INTRODUCTION}

Le Droit de la famille en Afrique renferme une particularité qui fait de lui un Droit à part entiers malgré l'apport de l'influence du Droit moderne.

L'Afrique a été l'une des terres fertiles d'exportation du Droit et même des juristes occidentaux, autant par l'invasion physique que par l'invasion intellectuelle, et il n'est pas inopportun de se demander si, à l'heure actuelle, les systèmes juridiques du «continent noir» sont encore liés par le nombril à ceux des anciennes puissances coloniales.

Depuis « l'arrivée des Blancs» en Afrique, le continent noir a été marqué par un phénomène original de pluralisme juridique où se mêlent Droits authentiquement locaux et Droits importés de gré ou de force, qu'il s'agisse des Droits religieux ou du Droit dit moderne. Dans un tel contexte de constante cohabitation ou de confrontation continue entre coutumes ancestrales et règles occidentales, il ne semble pas superflu de consacrer de nouvelles réflexions sur l'état et le devenir des Droits négro-africains à travers leurs diverses sources d'influence'.

Malgré les normes imposées par les législateurs en Afrique, il se manifeste une certaine résistance des pratiques coutumières qui font que ces normes ne semblent pas s'adapter aux réalités vécues dans les sociétés traditionnelles.

Tel est le cas du Droit successoral, qui, à un certain niveau ne s'adapte pas aux prescrits légaux au regard de la conception qui ressort de la particularité du partage héréditaire.

Nous citons par exemple la vocation successorale du conjoint survivant qui pose problème surtout lorsque celui-ci est la veuve.

Notre réflexion présente le rapport de la tradition et la modernité dans les Droits de la famille africains ensuite ressort l'existence d'un système juridique africain en matière successorale et enfin nous retenons le pluralisme juridique comme solution pour la compréhension du Droit de la famille en Afrique.

* Chef de Travaux à la Faculté de Droit de 1‘Université de Lubumbashi.

** Professeur Ordinaire à la Faculté de Droit de 1'Université de Lubumbashi.

1 THIOYE MOUSSA, « Part respective de la tradition et de la modernité dans les Droits de la famille des pays d'Afrique noire Francophone », in Revue internationale de Droit comparé, Vol.57, N57, 2005 , p.346. 


\section{A. Tradition et modernité dans les Droits de la famille}

\section{Le poids de la tradition}

Les Droits africains originels de la famille, qui sont d'une existence et d'une originalité certaine, demeurent plus que jamais vivaces même s'ils ont connu des agressions et des métamorphoses incontestables ${ }^{2}$.

Au-delà de la reconnaissance de l'existence de Droits originellement négro-africains, on s'accorde aujourd'hui à admettre que, malgré leur grande diversité, ces Droits peuvent tous être rangés dans un seul et même système ou famille juridique ${ }^{3}$.

L'existence de Droits typiquement africains est incontestable même si certaines circonstances ou caractéristiques particulières auraient pu et pourraient encore, de prime abord, en faire douter et ce, d' autant plus que pour « séparer chaque jour davantage l'Africain du monde qui lui est propre afin de l'acculturer au modèle occidental », on a entretenu « pendant longtemps en Europe l'illusion de l'inexistence des Droits africains qu'on avait vite fait de réduire à des usages ou à des coutumes sans force juridique $»^{4}$.

Les sociétés africaines ont effectivement connu et connaissent encore, tous au moins en fait, une technique législative permettant l'édiction, bien qu'orale le plus souvent, des règles de Droit de portée générale par l'autorité sociopolitique compétente (roi ou chef légiférant seul ou en conseil du fait du caractère souvent collectiviste du pouvoir; institutions diverses telles que sociétés secrètes ou classes d'âge; assemblées populaires, etc.) $)^{5}$.

Il faut également souligner la place occupée, au sein des sources juridiques traditionnelles, par la jurisprudence, modalité de formulation (orale) du Droit par une autorité chargée de trancher les contestations ${ }^{6}$.

Il convient, enfin, de rappeler le rôle de la doctrine en tant que mode de création du Droit sous la forme d'opinions exprimées par des dépositaires, bien définis ou non, des traditions du groupe ${ }^{7}$.

\section{Le Droit de la famille, une réalité malgré le pluralisme normatif.}

Les règles juridiques ne sont pas les seules qui régissent et régulent la vie en société même si ces dernières sont souvent perçues comme celles « dont la stricte observance garantit l'avènement de l'ordre social souhaité [...], parce qu'elles sont seules assorties de

2 NDOKO NICOLE CLAIRE, « Les manquements au Droit de la famille en Afrique Noire », in Revue Internationale de droit comparé, volume 43, n 1, Yaoundé, 1991, p.44.

3 THIOYE MOUSSA, op. cit., p.350.

4 Ibidem.

5 THIOYE MOUSSA, op. cit., p. 349.

6 DRA AMPARO MONTANANA, "La veuve et la succession héréditaire dans le droit classique », in Revue de droit international des Droits de l'antiquité, serie3, Paris, 2000, p.421.

7 AMSTOU SOW-SIDIBE, Le Pluralisme juridique en Afrique : l'exemple du droit successoral sénégalais, L.G.D.J, T1, Paris, 1991, p.456. 
contrainte et de sanctions organisées par l'État $\rangle^{8}$. D'autres systèmes normatifs (morale, mœurs, politesse, civilité, honneur, etc.) y contribuent dans des proportions équivalentes voire supérieures.

Il se crée entre les différentes catégories de normes sociales une situation de concurrence ou de complémentarité, une situation que tend à souligner la théorie du pluralisme normatif (à ne pas confondre avec le pluralisme juridique). Or, il semble que, dans le cas particulier de l'Afrique Noire, cette complémentarité est doublée d'une forte interpénétration des normes de sorte qu'il y aurait une grande difficulté, voire une impossibilité (accentuée par le fait que les coutumes font office de source essentielle des Droits originels) à distinguer nettement les normes dotées de juridicité pure et celles qui, strictement, en seraient dénuées. « La plupart du temps, en effet, les sociétés traditionnelles distinguent mal les règles religieuses, les règles juridiques, les règles de convenance, les règles d'hygiène, les règles techniques, les mythes de Genèse, les récits du passé, les proverbes, la sagesse des ancêtres, etc. $»^{9}$.

On a alors pu parler, face au trouble posé par « un Droit qui ne s'affirme pas autonome », de « coutume indistincte pour désigner cette nébuleuse normative » d'où sont sortis, « dans la suite de l'évolution des systèmes plus spécialisés, la religion, l'éthique, les mœurs, les manières de vivre et même le droit ${ }^{10}$.

En atteste, si besoin en est, l'intérêt que les juristes portent depuis quelques années aux Droits «traditionnels », après que ceux-ci ont été durablement la chasse gardée des ethnologues, sociologues et anthropologues (du droit tout au plus).

\section{Droit de la famille, une réalité malgré la relégation européocentriste à la primitivité11.}

Il n'est point nécessaire de se munir d'un microscope pour être en mesure de constater que les qualificatifs « traditionnels », « coutumiers », " ethniques », " primitifs »... ou 《 archaïques » sont souvent utilisés pour désigner les Droits originellement et authentiquement africains. On rencontre même, moins fréquemment, les expressions plus lourdement connotées de « droits arriérés », « Droits sauvages » ou « Droits barbares ».

Pourquoi en va-t-il ainsi, même lorsque les « droits indigènes » en question ont traversé les âges et sont restés vivants et vivaces? Pourquoi les oppose-t-on au Droit dit « moderne» qui, lui, est occidental et largement issu du Droit romain, et remonte à l'Antiquité? Il semble qu'il s'agit là d'une affaire d'ethnologie juridique puisqu'il n'est pas seulement question de simple chronologie (même si cette donnée est indiscutable), de simple différenciation entre des Droits anciens et un Droit plus récent. D'ailleurs, selon un critérium cultu-

8 THIOYE MOUSSA, op. cit., p.349.

9 AMSATOU SOW-SIDIBE, op. cit., p.362.

10 NDOKO NICOLE CLAIRE, Les manquements au droit de la famille en Afrique noire, RIDC, Yaoundé, 1991, p.47.

11 THIOYE MOUSSA, op.cit., pp.355 - 356. 
rel, les Droits africains sont, entre autres, dits traditionnels (coutumiers, primitifs, archaïques, sauvages, barbares...) seulement parce qu'il s'agit de « Droits étrangers à la civilisation de la majorité des ethnologues, étrangers à la civilisation occidentale, européenne, judéo-chrétienne... ». Autrement dit, ces Droits ne sont couverts du manteau de l'archaïsme qu'aux yeux de l'observateur occidental qui les perçoit ainsi, sous un angle européocentrique, même si ces droits constituent encore des normes positives et effectives d'un peuple ou d'une « peuplade $»^{12}$.

\section{B. L'existence d'un système juridique africain en matière successorale.}

La succession dans notre milieu urbain est une affaire essentiellement coutumière et par conséquent familiale.

A ce propos, il convient de rappeler que les congolais en particulier, comme tous les autres africains en général, admettent un système de double famille : la petite famille, d'une part et la grande famille, de l'autre. Le Droit successoral africain est organisé sur le fondement de cette conception dualiste ${ }^{13}$.

Alors que la diversité ou, parfois, la disparité des Droits originellement africains fait à priori douter de l'existence d'une véritable famille juridique, un regard plus attentif permet de répertorier des points communs et, partant, de conclure à l'existence d'un seul et même système juridique : Une diversité des Droits originels qui incline à opter pour la négative... Il est assez courant de parler de l'Afrique au pluriel pour en traduire, à juste titre, la diversité sur les plans ethnique, culturel, coutumier, linguistique, religieux, politique, etc. Cette diversité a d'ailleurs fait écrire que, « en réalité, à part la notion géographique, l'Afrique n'existe pas » puisque «l'Afrique, ce sont des milliers de situations les plus diverses, les plus variées, les plus contradictoires ». Il n'y aurait pas une Afrique, mais des Afriques tant est grande « la fragmentation qui caractérise les identités culturelles autochtones ${ }^{14}$.

On peut dire qu'il existe, en Afrique, plusieurs espaces juridiques, et non pas un seul, puisqu'on y rencontre de nombreuses sociétés globales (ethnies ou groupes humains) qui ne sont pas soumises aux mêmes Droits « traditionnels ». En effet, les Droits coutumiers ne se présentent pas de façon homogène en raison de la diversité culturelle et des spécificités régionales, sachant que les champs territoriaux d'application de ces Droits coïncident approximativement aux zones d'implantation des groupes ethniques soumis à ces Droits ${ }^{15}$.

Il s'ensuit, logiquement, qu'il n'y a pas un Droit traditionnel africain, un espace juridique africain, mais des Droits et des espaces juridiques africains dont le colonisateur a pu tenter de dresser un inventaire forcément incomplet. Ce qui n'est pas sans susciter, parfois,

12 NDOKO NICOLE CLAIRE, op.cit., p.45.

13 MUSANGAMWENYA WALYANGA, De la succession en droit civil congolais : problématique de la survivance des coutumes, Thèse de doctorat en Droit soutenue à l'université de Lubumbashi 2009, Inédit.

14 THIOYE MOUSSA, op.cit., pp. 355-356.

15 AMSATOU SOW-SIDIBE, op.cit., p.362. 
des conflits de Droit inter coutumier, que ce soit des antagonismes entre des personnes de coutumes différentes, des conflits consécutifs à des conversions (à une religion) ou brassages ou encore des conflits interrégionaux. En outre, cette diversité, donnant a priori une impression de désordre, a conduit les analystes à s'interroger sur l'existence d'un véritable système ou famille de Droits originellement africains ${ }^{16}$.

Derrière la diversité, il y a une organisation et une cohérence certaines, « un air de famille, des caractéristiques communes » qui révèlent une conception négro-africaine très originale du Droit. En effet, puisque le Droit présente un caractère relatif en raison des différences de conception que l'on peut en avoir et que l'on en a effectivement à travers le monde, il n'est guère surprenant de constater que les Négro-africains aient une conception de l'ordre social différente de celle des Occidentaux ${ }^{17}$.

En effet, les Droits originellement africains et le Droit moderne constituent deux systèmes de pensée juridique profondément différents, notamment par leurs origines, leurs finalités ainsi que par la place qu'ils accordent à l'individu au sein du groupe. S'agissant, en premier lieu, des sources qui sont généralement orales, les Droits coutumiers plongent dans le passé le plus authentiquement africain alors que le Droit moderne trouve son origine en Europe, dans les systèmes juridiques contemporains et, plus loin, dans la pensée politique grecque ainsi que dans la pensée juridique romaine. Les Droits dits traditionnels sont ainsi enracinés dans une philosophie particulière, une religiosité ou une cosmologie originale. S'agissant, en deuxième lieu, des finalités, on constate que les sociétés dites modernes «valorisent le progrès et l'innovation " tandis que les sociétés traditionnelles "valorisent le passé et mettent leur idéal à s'écarter le moins possible de ce passé qu'elles considèrent volontiers comme un âge d'or ${ }^{18}$.

Dans ces derniers groupements, la sécurité juridique se trouve, non dans la quête du changement (modernisation), mais dans la préservation des valeurs ancestrales considérées comme intemporelles ou presque (néanmoins, pour tenir compte des mutations) nouvelles au sein de la société, il y aurait des moyens de faire évoluer les droits coutumiers : S'agissant, en troisième lieu, du rôle dévolu à l'homme, la pierre angulaire dans les Droits dits traditionnels se trouve dans l'idée de communauté ou de solidarité (familiale, villageoise, tribale ou ethnique) ${ }^{19}$.

\section{Les Droits originellement africains entre constance et mutation}

La société et les Droits négro-africains originels (de la famille) ont traversé les âges et efficacement résisté aux invasions étrangères même s'il est incontestable prouvé qu'ils ont

16 THIOYE MOUSSA, op.cit., pp.357.

17 NDOKO NICOLE CLAIRE, op.cit., p.43.

18 NDOKO NICOLE CLAIRE, op.cit., p.43.

19 Ibidem. 
connu des mutations plus ou moins significatives ${ }^{20}$. Certaines racines restent ancrées dans le comportement de chaque membre, selon la société.

\section{Survivance et ténacité des Droits ancestraux.}

Régnant sans partage avant la période coloniale, la tradition a dû opposer au Droit moderne une farouche résistance pendante et même après l'occupation européenne. L' Exclusivisme anticolonial, dans l'Afrique Noire précoloniale s'est fait sentir. L'ordre et les rapports sociaux étaient, faute de droits infligés, exclusivement régis par les règles coutumières éventuellement islamisées. Il fallait, en effet, tenir compte des perturbations ou altérations apportées par l'Islam dont l'expansion en Afrique était une réalité dès le VIIe siècle. Ce déferlement de la religion musulmane, qui a commencé en Afrique du Nord avant de se poursuivre en Afrique orientale et occidentale, s'est accompagnée de la diffusion de son droit (ou de ses droits si l'on tient compte de la variété des écoles : malékite, hanéfite, chaféite et hanbalite). Il convient néanmoins de remarquer que, par le phénomène notamment du syncrétisme religieux, il s'est produit une négro-africanisation de l'Islam et, à l'inverse, une logique d'islamisation de certaines coutumes ${ }^{21}$.

Ce brassage est, du reste, tellement profond que les règles coutumières authentiquement africaines et celles islamisées sont souvent logées à la même enseigne, celle de droits dits traditionnels selon lecritère ethnocentrique occidental déjà évoqué.

Aussi évoquerons-nous les Résistances coloniales et postcoloniales. Ici, La Conférence de Berlin de 1885 avait ouvert l'ère officielle du partage de l'Afrique (à l'exception de l'Ethiopie et du Libéria déjà indépendants) entre les nations européennes : Allemagne, Belgique, Espagne, France, Grande- Bretagne, Italie et Portugal. Avec cette définition d'espaces coloniaux (revue suite aux deux conflits mondiaux), s'ouvrait par-là même, un processus d'implantation progressive, directe ou indirecte, forcée ou plus ou moins consentie, des droits et systèmes juridiques européens sur le continent africain dont les traditions étaient pourtant sensiblement différentes ${ }^{22}$.

Ce phénomène allait aboutir, sauf pour certains Droits privés régissant la vie quotidienne des Africains, à la substitution ou, plutôt, à une tentative de substitution d'un ordre légal nouveau au cadre juridique origine ${ }^{23}$.

Le contact entre pouvoir colonial et pouvoirs locaux produisit des chocs brutaux (d'autant plus que la transplantation des principes métropolitains, avec ou sans adaptation préalable, s'effectuait, le plus souvent, sans période transitoire) dans quasiment tous les domaines : politique, économique, social, culturel... et, bien évidemment, juridique avec l'imposition de textes fort exotiques à un milieu africain devenu, malgré lui receveur. Il y eut,

\footnotetext{
20 Ibidem.

21 THIOYE MOUSSA, op. cit., pp. 355-356.

22 Idem.

23 AMSATOU SOW-SIDIBE, op. cit., p.461.
} 
en effet, « un phénomène de mutation sur le plan du Droit qui est à la fois considérable et radical, qui constitue un véritable déracinement de cultures juridiques séculaires $»^{24}$.

\section{La part sans cesse grandissante de la modernité.}

Par le phénomène dit de "migrations de systèmes juridiques », les Droits originellement africains sont, depuis longtemps et aujourd'hui encore, sensiblement concurrencés sur leurs terres par le Droit dit moderne. En effet, il s'est produit en Afrique une opération de transplantation du Droit occidental, une transplantation qui, initialement faite par voie d'imposition, s'est poursuivie par voie de réception plus ou moins volontaire ${ }^{25}$.

Depuis les indépendances, les États africains sont confrontés, de façon récurrente, voire rémanente, à des discussions théoriques (au sommet) et pratiques (à la base) sur l'épineuse question du modèle de pensée ou de politique juridique à adopter. Comment dénouer le conflit désormais interne, hérité de la colonisation, entre les Droits originellement africains et le Droit dit moderne?

Comment et dans quelle mesure, faut-il satisfaire les revendications d'authenticité (retour aux sources en maintenant exclusivement les droits ancestraux) et/ou celles de modernité (occidentalisation à outrance du droit étatique)?

Aux voies extrêmes empruntées ou proposées par certains, faudra-il préférer les pistes médianes suivies ou suggérées par d'autres? ${ }^{26}$

\section{Alternative rigide entre tradition et modernité.}

Avec les indépendances, les tout nouveaux États africains qui avaient recouvré la liberté s'étaient trouvés, pour la plupart, confrontés à un problème de choix (de politique juridique et, même, de politique tout court) entre deux tendances contradictoires : d'une part, par le retour pur et simple aux traditions ancestrales en rayant d'un trait de plume l'héritage colonial et, d'autre part, le reniement des institutions traditionnelles au profit d'une modernisation ou occidentalisation à outrance ${ }^{27}$.

\section{La voie irréaliste de l'occidentalisation à outrance.}

Le développement du système juridique (inscrit dans le sillage du développement économique et social) passe par une identification complète au système moderne et, corrélativement, par une « condamnation à mort » des droits originellement africains. Le Droit occidental, hérité ou inspiré de l'ancien colonisateur, est considéré comme le seul en mesure de permettre la réalisation de certains objectifs primordiaux : volonté de mise en place d'un

24 NDOKO NICOLE CLAIRE, op.cit., p.45.

25 THIOYE MOUSSA, op. cit, pp. 355-356.

26 Idem.

27 Ibidem. 
État unitaire et fort, volonté d'assurer le développement économique (contre lequel certaines coutumes seraient un frein ou un obstacle), volonté d'unification et d'égalisation des statuts (mettre tous les citoyens sous le même Droit, en supprimant les discriminations raciales, ethniques, religieuses, sexistes...), etc. Tout cela fait que l'on se trouve en présence d'un « État (législateur et juge) contre le droit traditionnel $»^{28}$.

\section{Les voies médianes: Syncrétisme ou concurrence entre tradition et modernité}

La solution nous semble être dans un syncrétisme qu'il faudra opérer du système juridique traditionnel avec celui moderne ${ }^{29}$.

C'est donc la mise en place d'un pluralisme juridique qui ferait coexister sur des voies parallèles et concurrentes (chaque élément gardant son individualité ou son identité), un Droit traditionnel reconnu par les institutions officielles et un autre, moderne importé : ainsi, le Droit étatique permet et prévoit clairement qu'une même question juridique puisse être réglée, au choix des intéressés, soit par le Droit coutumier, soit par le Droit reçu de l'extérieur. Il en résultera une transaction certaine, plus ou moins équilibrée, entre la tradition et la modernité qu'un retour radical à celle-là ou une orientation brutale vers celle-ci ${ }^{30}$.

4. De la fusion ou unification des systèmes juridiques traditionnels et moderne comme une solution efficace.

Pour un certain nombre d'États africains, la voie de l'unité nationale passe nécessairement par celle de l'unification du Droit qui, d'une part, s'attaque aux particularismes locaux symbolisés par les Droits des divers groupes ethniques enfermés dans les frontières de l'État moderne et qui d'autre part, s'efforce de concilier tradition et modernité ${ }^{31}$.

L'intégration des Droits revêt donc un double aspect : d'une part la réduction à l'unité des différences entre Droits originellement africains et de l'autre la synthèse de ceux-ci avec le ou les Droits importés, qu'ils l'aient été avant ou pendant l'époque coloniale. L'idée est donc celle d'une fusion ou unification qui, excluant tout pluralisme juridique, s'efforce de couler le Droit coutumier et le Droit moderne dans une seule et même voie ${ }^{32}$.

Opposer le Droit traditionnel au code de la famille en matière de la dévolution héréditaire, a une incidence sur la conception de la famille. Cependant, le législateur colonial avait sérieusement rongé cette organisation en cherchant à faire triompher à tout prix la conception restreinte de la famille pour en tirer par la suite des conséquences sur le plan successoral $^{33}$

28 NDOKO NICOLE CLAIRE, op.cit., p.42.

29 THIOYE MOUSSA, op.cit., pp. 355-356.

30 NDOKO NICOLE CLAIRE, op.cit., p. 45.

31 Idem.

32 THIOYE MOUSSA, op. cit., pp.394.

33 MUSANGAMWENYA WALYANGA, op.cit., Inédit. 
Il ne s'agit pas, ici, de ressusciter purement et simplement le passé ou de reconduire sans l'altérer le Droit moderne. Il y a, certes, une sorte de reconduction des Droits ancestraux, mais ceux-ci doivent être activement repensés pour qu'ils soient en harmonie avec le contexte actuel qui a sensiblement évolué par rapport au contexte initial. De même, les modèles juridiques européens maintenus, devront être teintés d'une couleur local, ou « tropicalisés », ou plus péjorativement « cannibalisés ». De la sorte, on bâtirait des Droits de synthèse, métis, ouverts, non unilatéralistes, non uniformistes et, en définitive, des Droits entrés dans «l'âge du donner et du recevoir ». Il y aurait ainsi « adoption de lois nouvelles destinées à fondre les éléments de Droit traditionnel dans le Droit moderne et rendues applicables à l'ensemble de la population $»^{34}$.

Cette « volonté de l'Afrique de demeurer elle-même pour et par la sauvegarde des valeurs de civilisation traditionnelles, tout en s'ouvrant pour le progrès matériel aux influences extérieures », ne chercherait-elle pas à moderniser les Droits africains sans véritablement les dénaturer ou les occidentaliser? Ne serait-ce pas vouloir tout avoir, avoir « tout en un »? Entreprise de métissage ou de co-intégration probablement illusoire, du moins en Droit de la famille, puisqu'il est déjà avéré, dans ce domaine, que « les Droits traditionnels et le Droit français n'ont pas réalisé cette heureuse symbiose qui aurait pu doter l'Afrique noire d'un Droit original dans lequel l'Europe serait présente sans exiger l'absence de l'Afrique $»^{35}$.

Tout cela pour dire, plus sérieusement, que la coexistence, la concurrence ou la complémentarité entre le Droit coutumier, incarné par le «continent noir », et le Droit moderne, symbolisé notamment par la France, reste ouverte en Afrique noire, même si, parfois, le premier doit se contenter de vivre informellement, clandestinement, à l'ombre du second. En effet, la situation sur terrain fait état des influences diverses, le monde juridique africain étant exposé, depuis longtemps, à des vents de tradition et, en même temps, à des courants de modernité ${ }^{36}$.

Le mimétisme total des institutions juridiques occidentales, préconisé ou tenté, au nom du progrès ou de la modernité, par certains législateurs à « l'esprit non décolonisé », n’a pas pu et ne pourrait jamais prospérer en Afrique « où le ralliement aux conceptions occidentales du Droit n'est généralement que de pure façade $»^{37}$.

\section{Du pluralisme juridique en Droit successoral Congolais}

\section{Notion du pluralisme juridique}

Le pluralisme consiste à mettre en pratique et en parallèle, dans le système Juridique congolais, deux corps de règles, à faire coexister deux régimes juridiques différents pour le même

34 THIOYE MOUSSA, op. cit., p.396.

35 THIOYE MOUSSA, op. cit., p.396.

36 Idem, p.394.

37 Idem p.395. 
problème de la dévolution successorale. C'est un pluralisme plus matériel que formel puisque, la loi, elle-même, l'organise. Le pluralisme en question découle de la constatation de faits qui constituent les éléments donnés de l'élaboration du Droit ${ }^{38}$.

Les questions du pluralisme juridique ou normatif, voire d'une approche pluraliste de la juridicité, sont au cœur des démarches de l'anthropologie du droit qui, si elle n'en a pas le monopole, leur donne néanmoins un caractère plus radical que d'autres théories et approches scientifiques du Droit ${ }^{39}$.

Perçue de prime abord comme une évidence, la plupart des approches critiques du Droit prennent ce pluralisme comme point de départ de leurs analyses afin de mieux le comprendre. A son origine, il se confond avec l'anthropologie du Droit, sauf que celle-ci se heurte, dès le départ, à un paradoxe dû au fait qu'elle s'intéresse à des cultures autres, c'està-dire des cultures qui ne partagent pas la matrice culturelle occidentale moderne. ${ }^{40}$ Dans nombre d'entre elles, le concept « Droit », tout comme les concepts qui lui sont attachés tels que l'Etat, le système juridique, le code, la personne juridique n'existent pas. Même les notions sous-jacentes comme écriture, individu, raison n'y existent pas $^{41}$.

A propos de la définition du pluralisme juridique, plusieurs tendances s'affrontent. Nous nous intéressons ici à celle qui fait la distinction entre la loi, en tant qu'œuvre d'un pouvoir politique différencié, lequel peut prendre la forme étatique, et la coutume, définie comme un ensemble de règles propres à un groupe social. Tout en reconnaissant à tout groupe social un pouvoir normatif lui permettant de régir ses rapports internes, cette première tendance se refuse à assimiler ce pouvoir à un système juridique. Elle estime que les normes particulières à un groupe reproduisent le plus souvent celles d'un droit applicable à tous les groupes ${ }^{42}$. Il s'agit là de la version faible du pluralisme juridique.

Dans sa version dite forte, le pluralisme part de l'idée que les différents groupes sociaux voient se croiser en leur sein une multiplicité d'ordres juridiques : le droit étatique, mais aussi celui que produit d'autres groupes, droits qui peuvent se rapprocher ou diverger. Se situant dans la version faible du pluralisme juridique ${ }^{43}$.

L'idée de pluralisme juridique africain suppose une « situation où une personne est en présence de différents ordres juridiques autonomes et selon ses choix, oriente la solution afin d'avoir un risque de conflit tant en ce qui concerne la juridiction compétente que le droit applicable. Jacques Vanderlinden définit alors le pluralisme juridique comme « l'exis-

38 AMSATOU SOW-SIDIBE, op.cit. p.22.

39 GHISLAIN OTIS, Méthodologie du pluralisme juridique, Editions Karthala, Paris, 2012 p.53.

40 GHISLAIN OTIS, op.cit., pp. 54 et 55.

41 Ibidem.

42 ROULAND, N.,, Anthropologie juridique, coll. « Droit fondamental», PUF Paris., 1988, p.84.

43 Ibidem. 
tence au sein d'une société déterminée, de mécanismes juridiques différents s'appliquant à des situations identiques» ${ }^{44}$.

\section{Contexte du pluralisme successoral en Droit congolais : cas du conjoint survivant}

Après une analyse du caractère que présente le Droit de la famille en Afrique en général et en République Démocratique du Congo en particulier, il sied à présent pour nous d'analyser dans quel contexte on rencontre le pluralisme successoral ou dans quel situation celui-ci se manifeste.

Le Droit successoral du conjoint survivant, particulièrement de la veuve, apparait clairement qu'il n'est pas seulement régi par la loi et qu'il subit l'influence de la coutume lorsque la veuve se retrouve en concurrence avec les membres de la famille de son défunt mari qui s'obligent à respecter certaines exigences coutumières.

Nous pensons néanmoins que pour assurer une certaine sécurisation des Droits de la veuve, le législateur doit prévoir certaines dispositions qui auront un soubassement coutumier, à l'exemple de celui qui caractérise la célébration du mariage.

Le Droit des successions, comme celui du mariage, offre une hypothèse de pluralisme juridique. Bien que ce concept soit encore imprécis à l'état actuel des recherches, le pluralisme juridique, de l'avis de Carbonnier, peut être considéré comme une constatation selon laquelle une diversité a envahi le milieu juridique ${ }^{45}$.

Le Droit des successions se caractérise aussi par cette diversité, parce qu'il n'est pas régi uniquement par une réglementation unique et globale, s'agissant de la part successorale de la veuve lorsqu'elle fait face aux exigences des membres de famille de son défunt mari.

Le pluralisme consiste à mettre en pratique et en parallèle, dans le système Juridique congolais, deux corps de règles, à faire coexister deux régimes juridiques différents pour le même problème de la dévolution successorale. C'est un pluralisme plus matériel que formel puisque, la loi, elle-même, l'organise. Le pluralisme en question découle de la constatation de faits qui constituent les éléments donnés de l'élaboration du droit ${ }^{46}$.

Comment alors comprendre et analyser un Droit qui est ignoré des sociétés dans lesquelles il existe? Comment parler de cultures juridiques sans surimposer sa propre vision sur elles? Comment comparer des expériences humaines qui, à priori, peuvent paraître incomparables? On voit pointer les enjeux du pluralisme :comment comprendre un phénomène à travers la diversité de ses manifestations en dégageant ses similitudes...et sans réduire le phénomène à l'unité?Comment définir le phénomène même, lorsque sa définition

44 VANDERLINDEN, J., Le pluralisme juridique. Essaie de synthèse, GILSEN J., (dir.), Le pluralisme juridique, Bruxelles, éd. de l'Institut de sociologie de l'Université de Bruxelles.,1972.pp1956.

45 CARBONNIER J. cité par AMSATOU SOW-SIDIBE, Le pluralisme juridique en droit Sénégalais des successions ab intestat, Thèse de doctorat en droit, Université de droit, d'économie et des sciences sociales de Paris, vol. I, (Paris II), 1987, p.16.

46 AMSATOU SOW-SIDIBE, op.cit., p.22. 
initiale semble complètement inadéquate dans un nouveau contexte?Le Droit tel que nous le connaissons dans la tradition occidentale moderne ne serait-il lui-même qu'une manifestation d'un phénomène plus général, le phénomène juridique, le Droit ou la juridicité, cristallisé sous d'autres formes dans d'autres cultures? ${ }^{47}$ Le Droit, phénomène juridique, luimême ne serait-il un phénomène universel qu'à partir de la vision du monde qui l'a élaboré? Quelle implication cette relativisation a-t-elle sur notre conception même du Droit? ${ }^{48}$

Certains phénomènes, à l'avènement du Code de la Famille, ont également été des facteurs déterminants du pluralisme successoral en Droit congolais.

Il s'agit d'abord de l'hétérogénéité sociale qui caractérisait la population Congolaise. Une multiplicité de coutumes foisonne dans le pays. La plupart sont de la lignée patrilinéaire, et d'autres matrilinéaires, comme nous l'avons démontré ci-haut. C'est la réunion de ces différentes données qui a été prise en compte par le législateur dans la rédaction du Code de la Famille ${ }^{49}$.Et si, à titre exceptionnel, le législateur a, dans le Droit du mariage, maintenu, dans sa célébration, les coutumes ancestrales, il en est autrement en droit des successions.

Les coutumes congolaises, considérées pour certaines par les autorités étatiques comme un frein au développement, et donc inacceptables, ne rentrent pas dans les termes du pluralisme successoral, dans ce sens que le législateur interdit la pratique des coutumes contrat legem.

Les coutumes traditionnelles sont pourtant vivaces à l'heure actuelle, dans l'imaginaire d'une bonne partie de la population. Leur survivance pose le problème de l'inadéquation entre le Droit "voulu" par l'Etat et celui "vécu" par une fraction des citoyens ${ }^{50}$.

En tant que création du législateur, le pluralisme successoral doit apparaitre également et fondamentalement comme une œuvre de technique juridique dans la recherche de cette unification du Droit moderne et traditionnel.

Il suffit seulement de voir dans la pratique quelques cas qui montrent que la place de la veuve, dans les successions, est influencée par l'avis de la famille de son défunt mari qui revendique l'application de la tradition selon laquelle les biens du défunt restent en famille. Que de fois nous avons assisté aux scènes où les père et mère, frères, sœurs du de cujus reprennent les biens de leur frère ou enfant décédé.

Mais, avec la loi portant code de la famille suscitée, la matière des successions a cessé d'être soumise à la coutume, aux textes épars et à la jurisprudence qui a marqué la période allant de 1960 à 1987 en l'absence d'une législation appropriée. Cette loi a donc mis un terme à la dualité qui a caractérisé le droit des successions dans notre pays depuis la coloni-

47 Séries Juridicités, Paris, Karthala, coll. Cahiers d'anthropologie du droit cité par GHYSLAIN OTIS, op.cit., p. 55.

48 GHYSLAIN OTIS, op.cit., p.55.

49 Voir à ce titre exposé des motifs de la loi n $16 / 008$ du 15 juillet 2016 modifiant et complétant la loi $\mathrm{n}^{\circ} 087-10 \mathrm{du} 1^{\mathrm{er}}$ août 1987 portant code de la famille.

50 AMSATOU SOW-SIDIBE, op.cit., p.25. 
sation. Mais, au regard de la recrudescence des problèmes successoraux sur la veuve, le code de la famille tel que modifié en 2016 n'a pas fait allusion à cette survivance de la coutume sur le cas de la veuve. Seules dorénavant les règles contenues dans le code de la famille sont d'application et s'imposent indistinctement à tous les congolais, en matière des successions. Ce sont des règles de droit commun. L'application de ces règles concourt à la sauvegarde des intérêts des héritiers et au maintien de l'ordre public ${ }^{51}$.

Mais au regard de tous ces problèmes que le Droit successoral suscite quand le conjoint survivant est une veuve, comment va-t-on préparer cette unification du code de la famille qui reste la seule loi applicable en République Démocratique du Congo en matière successorale? Faut-il chercher à y introduire des considérations des coutumes comme il en est pour le mariage ou faudra-t-il faire évoluer l'article 758 du code de la famille par rapport à la protection de la veuve.

Tout cela fait qu'on se retrouve devant un Droit successoral de la veuve qui est légiféré en sa faveur, mais qui rencontre une certaine résistance quant dans son application à certains milieux.

Dans ces conditions, force est d'admettre qu'en Afrique le pluralisme juridique a deux termes essentiels, l'un traditionnel, l'autre moderne, est la seule piste réaliste et que la voie de l'uniformité est un genre de perfection qui saisit quelquefois les grands esprits et frappe infailliblement les petits ${ }^{52}$.

Notre démarche s'oriente et s'inscrit dans cette optique au regard du caractère hybride que présente le Droit successoral Congolais.

La part de la veuve, comme nous l'avons présenté, est soumise à une forte contestation lorsque la famille ayant le lien de sang avec le de cujus est aussi appelée à succéder à celuici. Il nous faudra voir comment mettre en place un Droit successoral du conjoint survivant qui chercherait à unifier le Droit positif congolais confronté à cette continuité, survivance, persistance....des pratiques coutumières que nous avons relevées en vue d'assurer une sécurisation judiciaire et juridique de la veuve en Droit congolais.

\section{CONCLUSION}

Notre démarche vise à rapprocher le Droit traditionnel au Droit étatique qui est le Droit positif et consiste en la considération plurale du droit successorale. En d'autres termes, seul le Droit étatique doit être appliqué.

En effet, « il n'existe pas, dans les branches du Droit congolais, des lois novatrices à

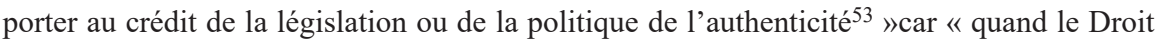

51 MUPILA NDJIKE H.F. K., Les successions en droit congolais, éd. Pax Congo, Kinshasa, 2000, p.27.

52 THIOYE MOUSSA, op.cit., p. 397.

53 BOSHAB E., « L'authenticité Zaïroise et l'interdiction de porter des noms aux consonnes étrangères, bilan de désuétude d'une loi pénale ", in Revue de Droit africain, Kinshasa, janvier 1999, pp. $35-37$. 
n'accède pas aux consciences et aux réalités psychologiques, l'homme ne l'accepte pas, ne s'incline pas devant ses impératifs, ne consent pas aux sacrifices qu'exige son application $^{54} » . \mathrm{Si}$ « la société, dit-on, est une cire molle que le législateur pourrait modeler à son gré $^{55}$ » à la fin, « lorsqu'on observe l'attitude de la population congolaise vis-à-vis des dispositions des codes, notamment du code de la famille, on constate qu'elle frise l'insurrection $^{56} \gg$.

Et pourtant, les partisans de l'unification forcée et autres modernisateurs du Droit africain savaient que même Portalis déclarait qu'en somme, pour être effectif, le Droit tire sa légitimité dans le peuple qui veut lui obéir, il doit être reconnu et accepté par ses destinataires $^{57}$.

Mais pour considérer un Droit successoral plural, certains éléments doivent être pris en compte au regard de la diversité culturelle de la population congolaise.

Présentant la situation juridique à Kinshasa, en République Démocratique du Congo, J.Pauwels a recherché à mettre en lumière l'importance du phénomène juridique urbain au Congo, et a souligné l'intérêt pour le juriste et les autres personnes concernées par le développement de la société congolaise de ne pas se contenter d'étudier les Droits coutumiers traditionnels ${ }^{58}$.

A cause de ce fait, Pauwels préconise la promotion d'un Droit uniforme qui impliquerait donc la création d'un nouveau corps de Droit. D'ailleurs, l'administration locale avait dès le début caressé le rêve de constituer pour les habitants africains de Kinshasa, un Droit moderne très semblable au droit occidental. Aussi la création d'un droit local nouveau a-telle été l'une des caractéristiques les plus marquantes de la vie juridique à Kinshasa ${ }^{59}$.Parlant des sources du Droit Kinois, on peut donc dire que celui-ci, au-delà de ses influences occidentales, est incontestablement resté un droit africain. Ce « Droit urbain », malgré son caractère fortement acculturé, s'adresse à des africains ${ }^{60}$.Tous les centres urbains rencontrent les mêmes problèmes. C'est pourquoi, nous pensons que dans la recherche d'éléments à tenir compte pour une considération du Droit plural, cette position de Pauwels est soutenable, car en matière successoral ce sont les mêmes difficultés qui se rencontrent dans tous les milieux.

La considération plurale vise dans le chef du législateur congolais à imposer dans la refonte du code de la famille les éléments qui fondent le Droit coutumier en matière successo-

54 BEKART H., Introduction à l'étude du Droit, Bruylant, Bruxelles, 1969, p. 165.

55 RANGERON F., Réflexion sur l'effectivité du Droit. Les usages sociaux du Droit, PUF, Paris, 1989, p. 139.

56 BOSHAB, E., op.cit, p. 52.

57 BEIGNIER BERNARD, «Portalis et le Droit naturel dans le code civil », in Revue d'histoire des facultés de Droit, Paris 1988 p.79.

58 PAUWELS J., « Le droit urbain de Kinshasa » in Congo-Afrique $n^{\circ} 25$ Kinshasa 1968., p. 253.

59 PAUWELS J., op.cit., p.253.

60 Idem. 
rale afin d'élaborer une législation acceptable par tous, comme préconisée dans l'exposé des motifs du code de la famille.

Afin de légitimer un Droit de la famille de type plural où les coutumes, les normes, les valeurs, les principes et la vision du monde des peuples autres que les Occidentaux sont incorporés dans un corpus juridique. Cela constituerait un modèle juridique représentatif de la diversité humaine, qui contribuerait davantage à faire régner l'harmonie dans la famille.

Ceci nous pousse à adopter une conception objectiviste selon laquelle « le Droit est donc ancré dans la réalité sociale; s'il existe, c'est parce que la société existe; il ne présente aucune transcendance, aucune autonomie par rapport à la société dont il reflète nécessairement les aspirations; il n'a, à vrai dire, aucune réalité en dehors de la vie collective $»^{61}$.

\section{BIBLIOGRAPHIE}

\section{TEXTES LEGAUX}

1. Loi n ${ }^{\circ} 006 / 06$ du 18/02/2006 portant Constitution de la République Démocratique du Congo telle que modifiée par la Loi n¹1/002 du 20 janvier 2011 portant révision de certains articles.

2. Loi n ${ }^{\circ} 71-002$ du 12 Juin 1971 portant création de la commission de réforme et d'unification du Droit civil Zaïrois.

3. Loi n 15/013 du 01 Août 2015 portant modalités d'application des droits de la femme et de la parité.

4. Loi $\mathrm{N}^{\circ} 16 / 008$ du 15 juillet 2016 modifiant et complétant la loi $\mathrm{N}^{\circ} 87-010$ du $1^{\text {er }}$ Août 1987 portant code de la famille.

\section{OUVRAGES}

1. AMSATOU SOW SIDIBE, Le Pluralisme juridique en Afrique : l'exemple du droit successoral sénégalais, L.G.D.J, T1, Paris, 1991.

2. BEKART.H, Introduction à l'étude du Droit, Bruylant, Bruxelles, 1969.

3. CARBONIER J., Droit civil : La Famille, P.U.F., Paris, 2004.

4. DUGUIT Léon, L'Etat, le Droit objectif et la loi positive, Dalloz, Paris, 2003.

5. KIFWABALA TEKILAZAYA, Droit Congolais : Régimes matrimoniaux, successions, libéralités, Les analyses juridiques, Lubumbashi 2013.

6. MUPILA NDJIKE K., Les successions en Droit congolais, éd. Pax Congo, Kinshasa, 2000.

7. OTIS GHISLAIN., Méthodologie du pluralisme juridique, Editions Karthala, Paris, 2012.

8. RANGERON F., Réflexion sur l'effectivité du Droit.Les usages sociaux du Droit, PUF, Paris, 1989.

9. ROULAND, N., Anthropologie juridique, coll. « Droit fondamental », PUF Paris., 1988.

10. VANDERLINDEN, J., Le pluralisme juridique. Essaie de synthèse, Le pluralisme juridique, éd. de l'Institut de sociologie de l'Université de Bruxelles.,1972.

61 DUGUIT LEON, L'État, le Droit objectif et la loi positive, Dalloz, Paris, 2003, p.8. 


\section{ARTICLES}

1. BEIGNIER BERNARD, «Portalis et le Droit naturel dans le code civil », in Revue d'histoire des facultés de Droit, Paris, 1988pp.78 - 101.

2. BOSHAB E. «L'authenticité Zaïroise et interdiction de porter des noms aux consonnes étrangères, bilan de désuétude d'une loi pénal », in Revue de Droit Africain, 9janvier 1999 pp.32 - 37

3. DRA AMPARO MONTANANA, "La veuve et la succession héréditaire dans le droit classique », in Revue de droit international des Droits de l'antiquité, serie3, Paris 2000 pp.415 - 448.

4. NDOKO NICOLE-CLAIRE, « Les manquements au Droit de la famille en Afrique Noire », in Revue Internationale de droit comparé, volume 43, $\mathrm{n}^{\circ} 1$, Yaoundé, $1991 \mathrm{pp} .87$ - 104.

5. PAUWELS J-M, « Le Droit urbain de Kinshasa » in congo-Afrique, ${ }^{\circ} 25$, Kinshasa, 1968 pp.239251.

6. THIOYE MOUSSA, « Part respective de la tradition et de la modernité dans les Droits de la famille des pays d'Afrique noire Francophone », in Revue internationale de Droit comparé, Vol.57, N ${ }^{\circ} 57,2005$ pp. $340-365$.

\section{THESE DE DOCTORAT}

1. MUSANGAMWENYA WALYANGA K.Gilbert, De la succession en droit civil congolais : problématique de la survivance des coutumes, Thèse de doctorat en droit soutenue à l'université de Lubumbashi 2009, Inédit. 